\title{
Ruptured pseudoaneurysm of superficial temporal artery
}

Sir,

Pseudoaneurysms are relatively rare vascular complications of closed head injury. The superficial temporal artery (STA) is the most frequent site of craniofacial traumatic pseudoaneurysm. Most of the cases present as expanding pulsatile masses along the course of the artery following a history of blunt injury to scalp. ${ }^{[1,2]}$ Rarely, it may increase in size to unacceptable cosmetic defect and rupture. The terminal branches of STA are the vessels most susceptible to injury. Although generally protected from trauma by the surrounding soft tissue, the branches of the STA sometimes approach the skin's surface in bony facial regions. At such locations, the branches are susceptible to injury by external forces.

A 70-year-old man presented with history of gradually progressive swelling over Rt temporal area for last 4 months and throbbing headache around the swelling for last 2 months. Swelling appeared following an incidence of blunt trauma to Rt temporal area 4 months back. Fifteen days back while taking bath, the swelling spontaneously ruptured with severe bleeding in a projectile manner, which was controlled with pressure bandage. On examination, the patient was conscious and well oriented to time, place, and person. His neurological examination did not reveal any abnormality. On local examination, we found a firm, nontender, globular pulsating swelling over Rt temporal area, of about $3 \mathrm{~cm}$ in diameter, with ulcer over its lateral aspect [Figure 1a]. The ulcer was covered with black eschar. Pressure over Rt temporal artery diminished pulsation of lesion. With this history and clinical finding, our provisional diagnosis was ruptured pseudoaneurysm of Rt STA. Patient was advised plain and contrast computed tomography (CT) scan of brain to exclude intracranial extension. Axial scan of brain reveal a soft tissue scalp swelling over Rt temporal area without underlying bone erosion or intracranial extension [Figure 1c]. Lesion

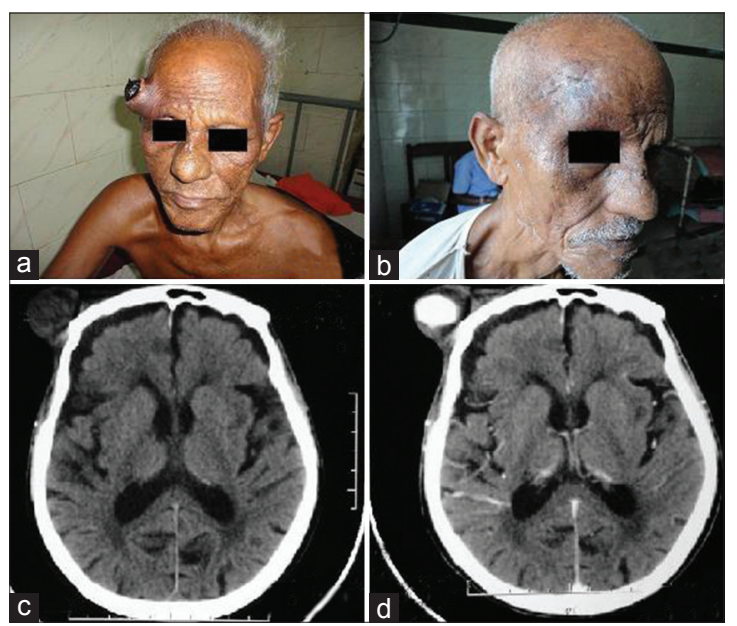

Figure 1: (a) Globular pulsating swelling over Rt temporal area of about $3 \mathrm{~cm}$ in diameter, with ulcer over its lateral aspect covered by black eschar. (b) Postoperative image after 15 days of surgery showing healthy scar and no skin necrosis. (c) Axial scan of brain reveals a soft tissue scalp swelling over Rt temporal area without underlying bone erosion or intracranial extension. (d) Axial scan contrast showing inner enhancing component of the STA pseudoaneurysm with surrounding soft tissue attenuation representing peripheral thrombus and adjacent inflammatory change 
showed contrast enhancement [Figure 1d]. We ligated STA proximal and distal to the lesion, followed by complete excision of it [Figure 2a and b]. Histopathologic study confirmed it as a case of pseudoaneurysm. Postoperative image after 15 days of surgery showing healthy scar and no skin necrosis [Figure 1b].

The first reported case of a temporal artery aneurysm was by Thomas Bartholin in 1740. ${ }^{[3]}$ Sporting injuries, temporomandibular joint surgeries, hair transplantation, and neurosurgical procedures have been implicated in its etiology. A true aneurysm is the one that involves all three layers of arterial wall (intima, media, and adventitia), whereas a false or pseudoaneurysm has a pseudocapsule consisting of mucopolysaccharide-rich connective tissue. It develops as a result of complete or incomplete disruption of arterial intima, followed by hematoma formation which is contained by the surrounding tissues. Gradually, a fibrous pseudocapsule consisting of mucopolysaccharide-rich connective tissue surrounds the hematoma. Further, hematoma gets lysed by circulating leukocyte, with eventual formation of a sac communicating with the artery. Successful endovascular obliteration of the STA aneurysm has been reported. However, ligation of the afferent and efferent vessels, with excision of the aneurysm, under local or general anesthesia, remains the treatment of choice. End-to-end
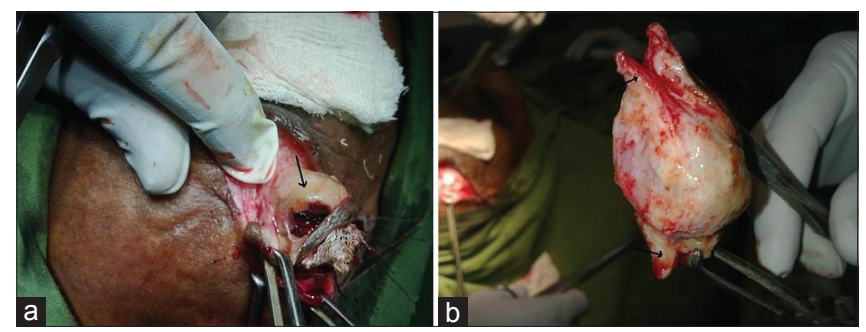

Figure 2: (a) Arrow showing pseudocapsule containing thrombus. (b) Arrow showing proximal and distal ends of STA. Pseudoaneurysm can be seen closely adherent to it anastomosis or arterial grafting is occasionally necessary to restore blood flow to critical dependent structures.

Although its incidence is very rare, surgeons should consider the possibility of a traumatic pseudoaneurysm in case a pulsatile palpable mass is found around preauricular region, following trauma. Most literature reports claim that STA pseudoaneurysmal surgery is commonly done for cosmetic reasons, but there is a potential risk of spontaneous rupture and life-threatening hemorrhage. In developing countries, this treatable condition can be neglected for a long time and can have serious consequences. Therefore, once diagnosed, it should be excised early to avoid spontaneous rupture and unacceptable cosmetic defect in future.

Sudhansu S. Mishra, Satya B. Senapati, Sanjay K. Behera, Acharya S. Pattajoshi, Deepak Das

Department of Neuro Surgery, SCB Medical College and Hospital, Cuttack, Odisha, India. E-mail: satya.bhusan.senapati@gmail.com

\section{REFERENCES}

1. Evans CC, Larson MJ, Eichhorn PJ, Taylor RS. Traumatic pseudoaneurysm of the superficial temporal artery: Two cases and review of the literature. J Am Acad Dermatol 2003;49: S286-8.

2. Han K, Borah GL. Pseudoaneurysm of the anterior superficial temporal artery. Ann Plast Surg 1996;37:650-3.

3. Schechter MM, Gutstein RA. Aneurysms and arteriovenous fistulas of the superficial temporal vessels. Radiology 1970;97:549-57.

\begin{tabular}{|l|l|}
\hline \multicolumn{2}{|c|}{ Access this article online } \\
\hline Quick Response Code: & Website: \\
\hline & www.ijns.in \\
\cline { 2 - 2 } & \\
\hline
\end{tabular}

